\section{Life Sciences HES-SO Valais/Wallis: Applied Research for Innovators}

Abstract: The Institute of Life Technologies at the HES-SO Valais/Wallis in Sion focuses on the areas of peptide and protein technologies, life and bioresource technologies, food and natural products and diagnostic systems. With this network of complementary areas of expertise, the institute can offer industrial partners a broad spectrum of integrated solutions.

Keywords: EPFL · HES-SO Valais/Wallis · Life sciences

\section{Strong in Peptide and Protein Technologies}

The Peptide and Protein Technologies research group focuses on the identification, characterization and development of peptides, peptidomimetics and proteins for therapeutic purposes, diagnosis and food applications. The group possesses the expertise and equipment required for the small-scale chemical and recombinant synthesis, analysis and production of peptides.

A typical example is the research on the Notch signalling pathway, a highly conserved cell signalling system that enables adjacent cells in the body of humans and animals to communicate with each other via the interaction between the Notch receptors on the surface of one cell and its transmembrane ligands on the surface of its neighbour. Notch is an important receptor in numerous developmental and differentiation processes in our body, for example in the formation of new blood cells or intestinal cells. Various illnesses, including numerous types of cancer, can form if this signalling pathway is impaired.

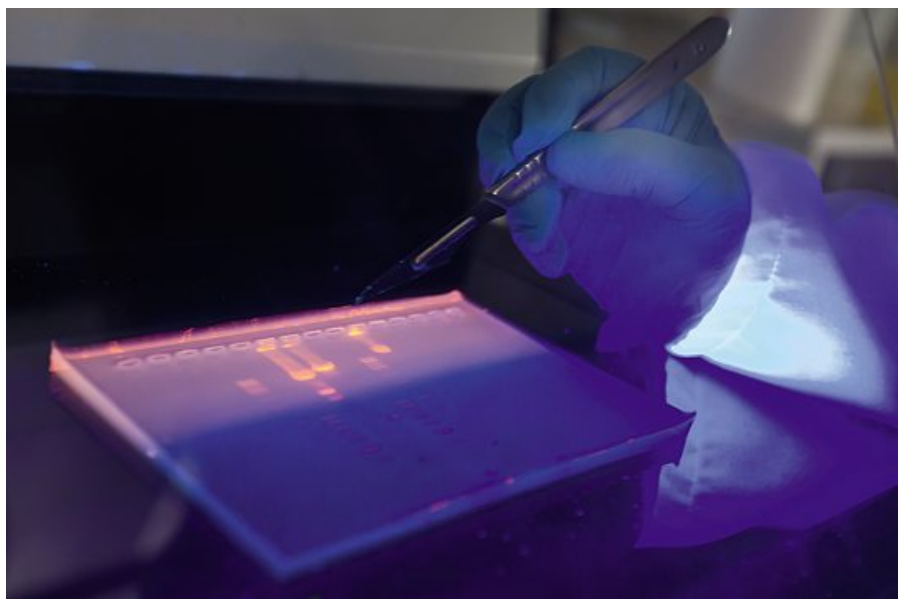

The Peptide and Protein Technologies research group focuses on the discovery and optimization of peptides and proteins for therapeutic, diagnostic and food-related applications. Photo: photo-genic.ch.

In order to open up this area for marketable products, the globally operating biopharmaceutical company Debiopharm, based in Lausanne, together with the Life Science faculty of EPF Lausanne, developed two bioassays for identifying inhibitors of the Notch signalling pathway. As a result EPFL, together with Debiopharm, the biotech firm Atheris, which specializes in the

\section{EPFL Campus Valais/Wallis - Impetus for Technology Transfer}

With the launch of the Campus Valais/Wallis in January 2014, EPFL has gained a foothold in the Swiss Rhone valley, joining forces with the HES-SO university, 'The Ark' foundation and the city of Sion. 11 new professorships are planned in the fields of energy, green chemistry - with biomass from agriculture and the conversion of $\mathrm{CO}_{2}-$ health with biotechnology and bioengineering, and nutrition. The latter involves a collaborative venture with SUVA, Sion Hospital and the Institute for Research in Ophthalmology (IRO). The budget of CHF 356 million covers a two-part building complex, one part for EPFL and its 150 researchers, and one for HES-SO Valais/Wallis. There will also be room for company start-ups and demonstrators, as well as the infrastructure for testing new energy systems. Marc-André Berclaz, economist and Chief Operating Officer (COO) of the Campus EPFL Valais/Wallis comments: "The linking of basic research at the EPFL with the applied research and development and industrial collaborations at the HES-SO will strengthen the value-added chain and allow synergies to be exploited." As the former President of the HES-SO and also of the Rectors' Conference of the Swiss Universities of Applied Sciences, he is familiar with the Canton of Valais, its political structures and the academic environment in Switzerland. "This project is progressing in stages and requires flexible and fluid processes. The aim is to stimulate entrepreneurial potential with the formation of start-ups, technology transfer between existing companies and the utilization of burgeoning innovations thanks to The Ark foundation". The relocation of HES-SO Valais/Wallis to the new campus is scheduled for 2018.

field of active molecules from animal toxins, and the molecular biology group at HES-SO Valais/Wallis headed by Prof. Sergio Schmid, initiated a project supported by the CTI, the Committee for Technology and Innovation. The project scientists will screen selected libraries in the two bioassays and thus identify various innovative peptides that inhibit the Notch signalling pathway. The aim of this collaborative venture is to optimize these peptides for future pharmaceutical research and to validate them in vitro and in animal experiments.

\section{Synthesis Gas Fermentation for Waste Recycling}

The Life and Bioresource Technologies research group focuses on topical social and economic issues. Examples include the recombinant production and chemical characterization of therapeutic peptides, proteins and antibodies, the recycling of food waste for energy generation, the biocatalytic conversion of substances in the microwave reactor or microbial fuel cell, and the generation of biopolymers for medical or industrial applications. Biopolymer generation is currently being investigated in the FP7 project SYNPOL (www.synpol.org) by Prof. Manfred Zinn and his team and 13 EU partners from industry and academia. The aim of the project is to link the pyrolysis of organic waste, e.g. municipal, commercial and agricultural waste and 
sewage sludge, with the production of biopolymers. "Since we recycle the generated synthesis gases such as $\mathrm{CO}, \mathrm{CO}_{2}$, $\mathrm{H}_{2}$, using bacterial fermentation we are relatively dependent on the composition of the organic waste. We are also seeking to include innovative, closed-loop process control systems in order to optimize the quality and quantity of the biopolymers", comments the biotechnologist, who specializes in bioprocess design and biomaterials. "Under defined conditions the bacteria produce polyhydroxyalkanoate, a starting material for biobased plastic prototypes that is ultimately extracted with solvents." The findings jointly acquired in the project will enable organic waste to be handled in an environmentally sound manner and thus reduce the harmful environmental effects of plastics.

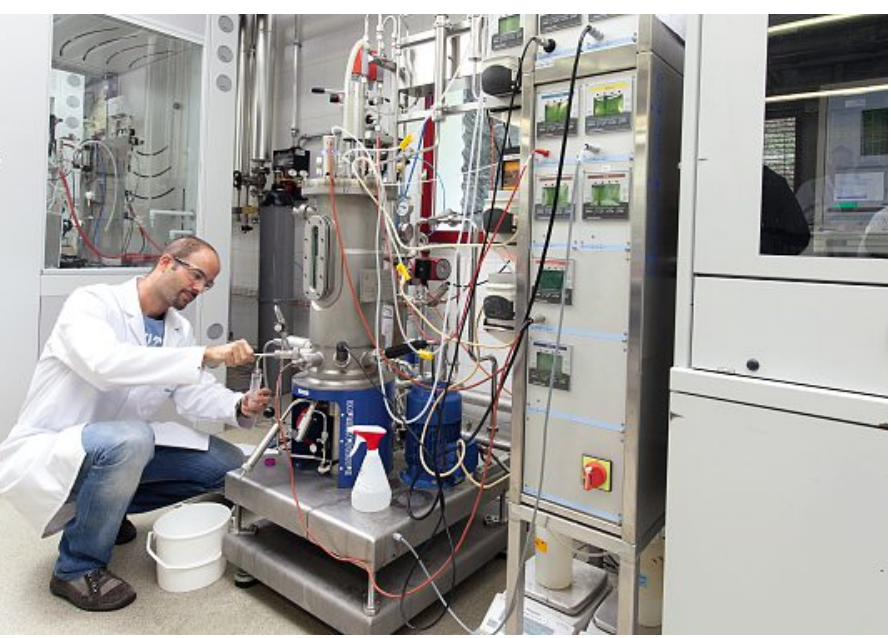

Fed-batch cultivation of Pseudomonas putida for the preparation of polyhydroxyalkanoates at HES-SO/Valais Sion. Photo: photo-genic.ch.

\section{Eating Healthily}

The interdisciplinary Food and Natural Products research group provides expertise in the processing of food and natural products, food microbiology, the safety of complete food chains and bioactive compounds and health. Working together with the University of Basel, they have developed the 'Master of Advanced Studies in Food Safety Management', a course that shows food safety inspectors and those responsible for control laboratories how to manage risk and crisis management successfully. "This is not a first-level university course, but rather - a rarity in Europe - a postgraduate university course of study eligible to those with a first degree and extensive professional experience",

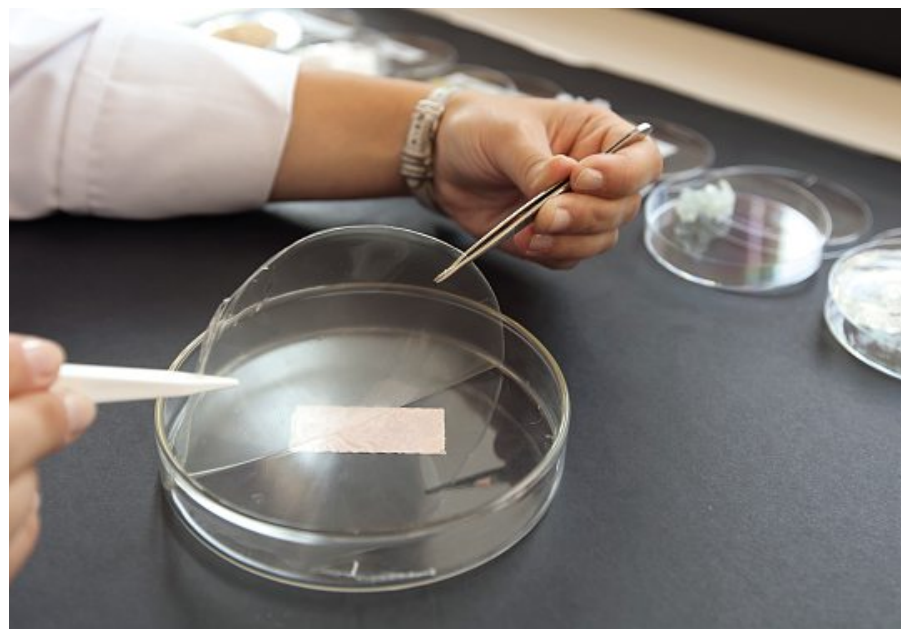

A new generation of bacterial biopolyesters is more transparent and possesses improved material properties. Photo: photo-genic.ch

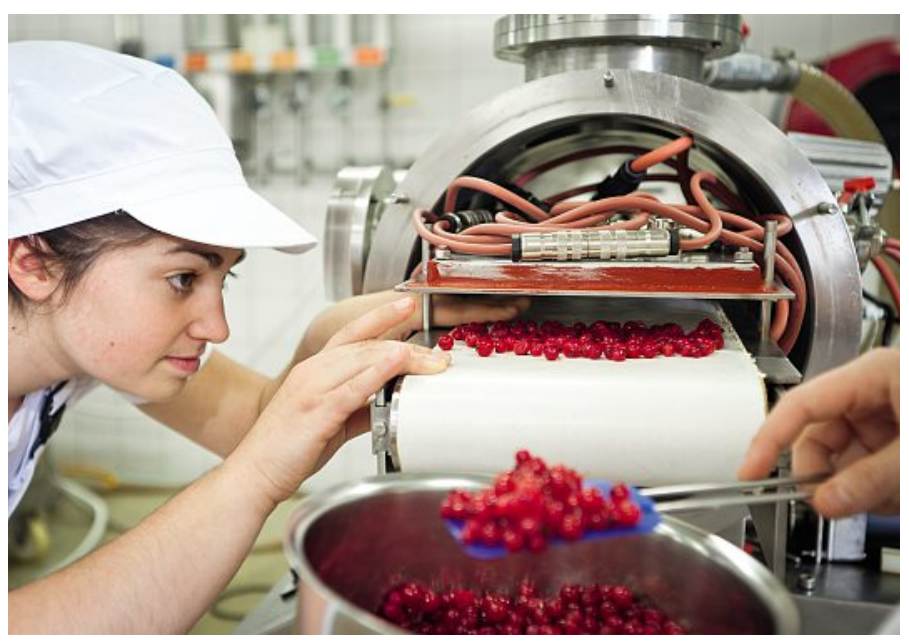

Quality assurance across the whole food chain poses a challenge for the Food Safety Manager. The vocational courses at the HES-SO convey knowledge relating to managerial tasks in food safety and food quality services. Photo: Céline Ribordy.

explains Prof. Margareta Neuburger, who has been responsible for further training at the University of Basel for over 20 years, developing a total of 31 postgraduate courses.

Food safety is a hot topic nowadays since consumers' trust has been severely eroded by incidents such as growth-promoting oestrogens in meat, the antifreeze glycol in wine and over 50 deaths and 3800 sufferers resulting from an EHEC (enterohaemorrhagic Escherichia coli) epidemic triggered by poor hygiene. So the subject of food safety has aroused considerable interest in those with a first-level degree and the necessary professional experience. According to Margareta Neuburger, "This is attributable to the fact that our course of study is certified and - provided all the performance criteria are satisfied - entitles the student to use an academic title." The study director Prof. Rudolf Schmitt has run the course twice since 2009 , in each case with 40 participants, and draws a positive conclusion: "With this course we are standardizing the training in Switzerland because we are offering just one course of study independently of the linguistic regions."

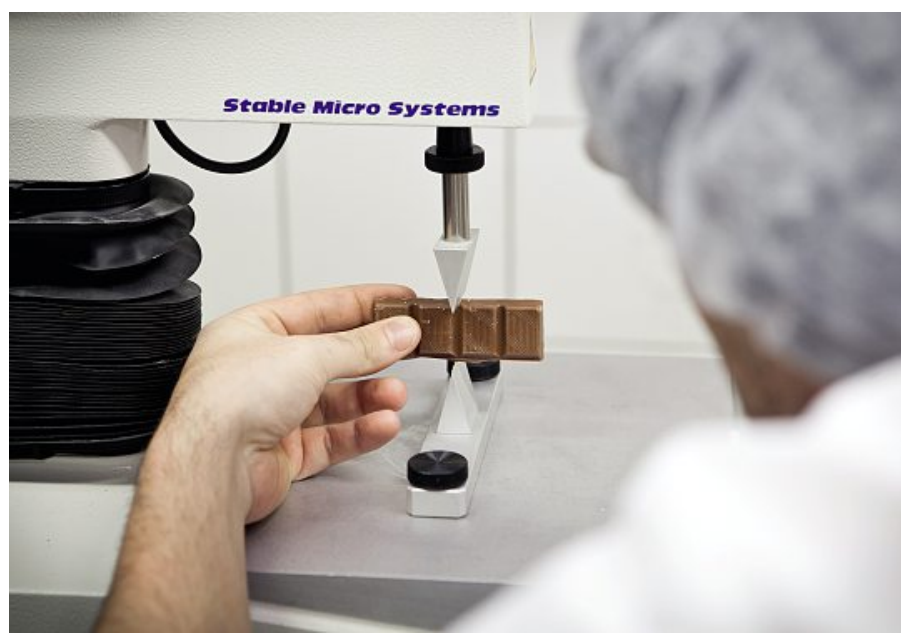

Legislators, standards organizations, retail companies and food producers are constantly developing new standards designed to improve food safety. Photo: Céline Ribordy.

\section{Joining Forces for Point-of-Care (POC) Diagnostics}

The Diagnostic Systems research group designs and develops innovative integrated solutions. The group currently focuses on cell systems, molecular diagnostics, phytoactive substances 
and point-of-care laboratory diagnostics (POC). In future it will be possible to detect and quantify, in real time, diagnostically relevant molecules in a circulating fluid such as blood or in an otherwise accessible local area of the body - for example during surgery. As a flagship project Prof. Marc Pfeifer mentions the development of a small and compact device for Therapeutic Drug Monitoring (TDM) in the doctor's office rather than, as is currently the case, in a central laboratory: "In the ISyPeM II nano-tera project funded by the SNSF (http://www.nano-tera.ch/ projects/368.php) EPFL is cooperating with HEIG-VD, CHUV in Lausanne and three institutes of the HES-SO, with the aim of improving TDM by reducing the required blood sample volume from millilitres to microlitres and by accelerating the reporting of results from hours or days to minutes", according to the expert in in vitro medical diagnostics. "In future, it will be possible to monitor the concentration of, for example, immunosuppressants, antibiotics or antivirals that need to be kept within a therapeutic window, conveniently in the doctor's office, instead of the patient having to visit the hospital."

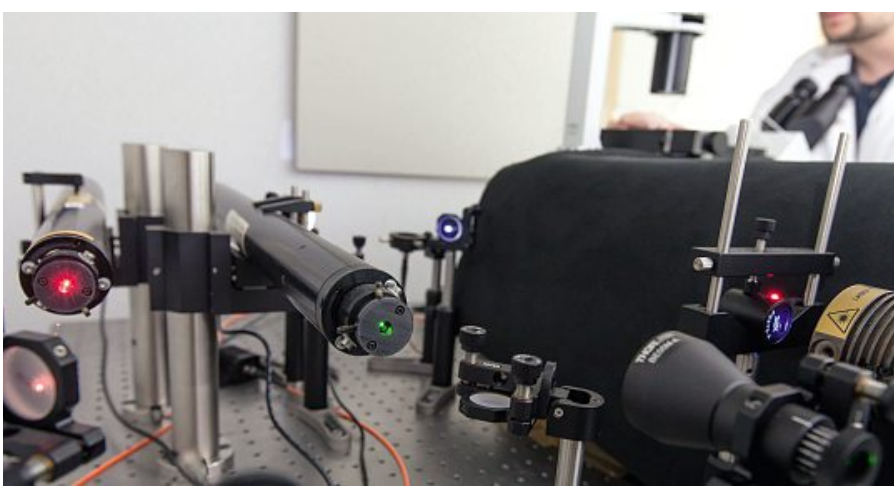

Optics laboratory at the HES-SO. The modern research equipment and commercial analytical instruments offer flexibility and comparison options in the development of point-of-care prototypes. Photo: Photo-genic.ch.

The combination of scientific expertise and industrial experience possessed by the researchers involved serves as a role model, since it allows numerous immunological, genetic and cellular assays, as well as analytical devices, to be developed within one organizational unit. "However," as Prof. Jean-Manuel Segura explains, "we must ensure that the various reagents, hardware and software components developed by the individual groups are compatible with each other when they are integrated in the prototype." On this project HES-SO Valais/Wallis acts as a 'one-stop-shop', delivering comprehensive solutions to customers. Accordingly, and in line with the 'bottom-up' principle, the Health Technology Innovation Center was established at the start of 2014. "By connecting the different institutes, the HTIC is able to tackle large, interdisciplinary projects", according to Michael Schuhmacher, coordinator of the HTIC and professor at the Institute of Information Systems. "Transfer of innovation to industry particularly makes sense if the developed solution also really meets the needs of end users and, at the design stage, takes account of important aspects such as cost-effectiveness, manufacture/production and regulatory requirements", adds HTIC vice-coordinator Marc Pfeifer.

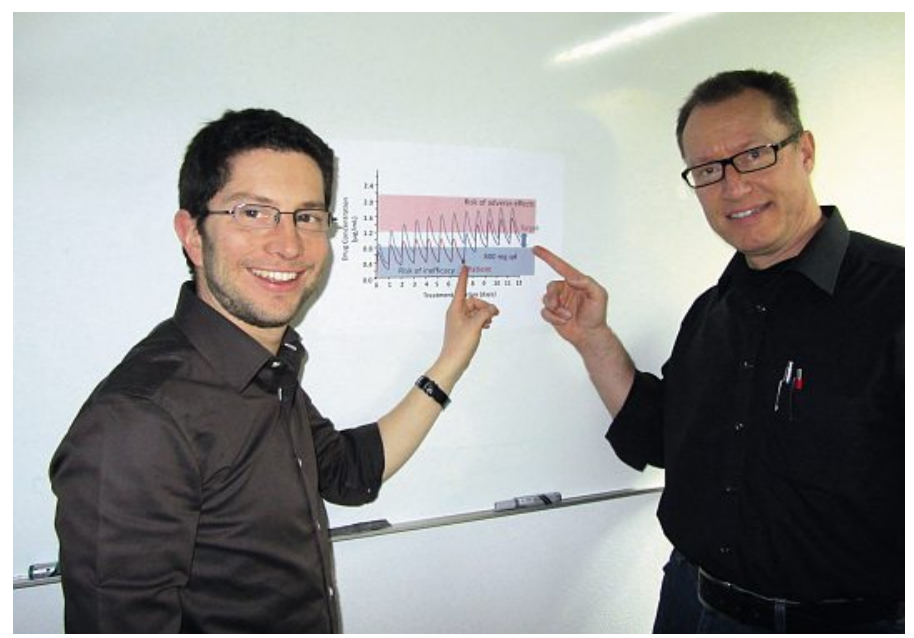

Prof. J.-M. Segura (left) and Prof. M. Pfeifer illustrate measurement points with sub-optimal and optimal drug concentrations respectively. Photo HES-SO.

With its peptide and protein technologies, life and bioresource technologies, food and natural products and diagnostic systems, the Institute of Life Technologies at HES-SO Valais/Wallis in Sion makes a valuable contribution to biotechnet Switzerland, enhancing Switzerland's position as a key hub for biotechnology internationally.

Elsbeth Heinzelmann

Science and technology journalist

Prepared on behalf of biotechnet

\section{Information:}

http://itv.hevs.ch/switzerland/institute-life-technologies.html www.foodsafety-mas.ch

www.b360.ch

Received: April 15, 2014 